\title{
On the removal of finite discrete spectrum by coefficient stripping
}

\author{
Barry Simon ${ }^{1}$
}

\begin{abstract}
We prove for a large class of operators $J$, including block Jacobi matrices, that if $\sigma(J) \backslash[\alpha, \beta]$ is a finite set, each point of which is an eigenvalue of finite multiplicity, then a finite coefficient stripped, $J_{N}$, has $\sigma\left(J_{N}\right) \subset[\alpha, \beta]$. We use an abstract Dirichlet decoupling.
\end{abstract}

Mathematics Subject Classification (2010). 47B26, 34L15, 81Q10.

Keywords. Coefficient stripping, block Jacobi matrices.

\section{Introduction}

The work in this note is motivated by the following theorem.

Theorem 1.1. Let $V \in L_{\mathrm{loc}}^{1}([0, \infty))$ be such that $-\frac{d^{2}}{d x^{2}}+V(x)$ is limit point at infinity. Suppose $-\frac{d^{2}}{d x^{2}}+V(x)$ with $u(0)=0$ boundary conditions on $L^{2}((0, \infty) ; d x)$ has finitely many eigenvalues (and no other spectrum) in $(-\infty, 0)$. Then there exists $A>0$ so that for all $a>A,-\frac{d^{2}}{d x^{2}}+V(x)$, with $u(a)=0$ boundary conditions on $L^{2}((a, \infty) ; d x)$ is a nonnegative operator.

The proof is an immediate consequence of Sturm oscillation theory: by hypothesis, the function with $u(0)=0, u^{\prime}(0)=1,-u^{\prime \prime}+V u=0$ has finitely many zeros. If $A$ is the last zero, $u \uparrow[a, \infty)$ solves $-u^{\prime \prime}+V u=0$ and has a definite sign on $[a, \infty)$. Thus, by Sturm comparison and oscillation arguments, the operator on $[a, \infty)$ is nonnegative.

While it appears that this argument is well known to some experts, I am aware of only two places that it appears explicitly in print: in Nikishin's lovely paper [2] and in my review article on Sturm oscillation theory for the Sturm 200th Birthday Conference [5].

Going back to Bôcher [1] (and conjectured to be known to Sturm), there are discrete analogs of Sturm oscillation theorems. One looks at zeros of the linear

\footnotetext{
${ }^{1}$ Supported in part by NSF grant DMS-0652919
} 
interpolation of the solution of the difference equations. This allows one to prove an analog of Theorem 1.1 for Jacobi matrices of the form

$$
J=\left(\begin{array}{cccc}
b_{1} & a_{1} & 0 & \cdots \\
a_{1} & b_{2} & a_{2} & \cdots \\
0 & a_{2} & b_{3} & \cdots \\
\vdots & \vdots & \vdots & \ddots
\end{array}\right)
$$

Here and below, $a_{n}>0, b_{n}$ is real, and $\sup \left(\left|a_{n}\right|+\left|b_{n}\right|\right)<\infty$. (Indeed, it was this case treated in [2] and [5]; Nikishin doesn't use the terminology "oscillation theorem" but he counts sign changes of certain determinants.)

Theorem 1.2. Suppose J given by (1) has finitely many eigenvalues outside $[\alpha, \beta]$. Let $J_{n}$ be the Jacobi matrix (1) with the top $n$ rows and left $n$ columns removed. Then for some $N$ and all $n>N, \sigma\left(J_{n}\right) \subset[\alpha, \beta]$.

Given the discrete Sturm oscillation and comparison theorems (for the linear interpolation), the proof of Theorem 1.2 is essentially the same as for Theorem 1.1.

The immediate motivation for this work is the question asked me by Rostyslav Kozhan: Does an analog of Theorem 1.2 hold for block Jacobi matrices, that is, objects like (1) but where for some $\ell$, the $b_{j}$ and $a_{j}$ are $\ell \times \ell$ matrices. Recently, Schulz-Baldes [3] has developed oscillation theorems for some block Jacobi matrices, and it may be possible to use his machinery to answer Kozhan's query. But we decided to seek out another and more direct approach. It has the advantage of working in cases where $a_{n}$ and $b_{n}$ are operators on an infinite-dimensional space where it is unlikely an oscillation theorem exists. Here is our main result:

Theorem 1.3. Let $J$ be a bounded selfadjoint operator on a Hilbert space $\mathscr{H}$. Suppose $\left\{P_{n}\right\}_{n=1}^{\infty},\left\{Q_{n}\right\}_{n=1}^{\infty},\left\{R_{n}\right\}_{n=1}^{\infty}$ are families of orthogonal projections that obey

$$
\begin{aligned}
& P_{n} Q_{n}=Q_{n} P_{n}=P_{n} R_{n}=R_{n} P_{n}=Q_{n} R_{n}=R_{n} Q_{n}=0, \\
& P_{n}+Q_{n}+R_{n}=1, \\
& \text { s- } \lim _{n \rightarrow \infty} Q_{n}=\underset{n \rightarrow \infty}{\mathrm{s}-\lim _{n}} R_{n}=0, \\
& P_{n} J Q_{n}=Q_{n} J P_{n}=0 .
\end{aligned}
$$

Suppose the only spectrum $J$ has in $(-\infty, 0)$ is finitely many eigenvalues of finite multiplicity. Then for some $N, Q_{n} J Q_{n} \geq 0$ for all $n \geq N$.

In the block diagonal case, we have the following result.

Corollary 1.4. Let $\mathscr{H}=\ell^{2}(\{1,2, \ldots\}, \mathcal{K})$, sequences with values in a Hilbert space, $\mathcal{K}$, with $\sum_{n=1}^{\infty}\left\|x_{n}\right\|^{2}<\infty$. Let $J$ be a matrix in $\mathscr{H}$ of the form (1) with entries $b_{j}, a_{j}$ that are bounded operators on $\mathcal{K}$ with $\sup _{j}\left(\left\|a_{j}\right\|+\left\|b_{j}\right\|\right)<\infty$. Suppose the 
only spectrum of $J$ in $(-\infty, 0)$ is finitely many points, each an eigenvalue of finite multiplicity. Then for some $N$ and all $n \geq N, J_{n} \geq 0$, where $J_{n}$ is $J$ with the top $n$ rows and left $n$ columns dropped.

Proof. Let $P_{n}$ be the projection onto $\ell^{2}(\{1, \ldots, n-1\} ; \mathcal{K}), R_{n}$ the projection onto $\ell^{2}(\{n\} ; \mathcal{K})$, and $Q_{n}$ the projection onto $\ell^{2}(\{n+1, n+2, \ldots\} ; \mathcal{K})$. (2) and (3) are obvious. (4) is simple and (5) is obvious. Thus, $J_{n}=Q_{n} J Q_{n} \geq 0$ for all large $n$ by the theorem.

We prove Theorem 1.3 in Section 2 and a continuum analog in Section 3.

We end this section with three remarks. (i) As noted in [5], theorems of the form of Theorem 1.3 imply that if $\sigma_{\text {ess }}(J)=[\alpha, \beta]$, then for any $\varepsilon>0$ and $n$ large, $\sigma\left(Q_{n} J Q_{n}\right) \subset[\alpha-\varepsilon, \beta+\varepsilon]$. This result is true under much weaker hypothesis (essentially, (5) is not needed), but I believe the stronger result that is Theorem 1.3 requires some kind of decoupling.

(ii) In Corollary 1.4 , one can take $\mathcal{K}=\ell^{2}\left(\mathbb{Z}^{\nu-1}\right)$ and so treat discrete Schrödinger operators on $\mathbb{Z}_{+}^{v}=\left\{\left(n_{1}, \ldots, n_{v}\right) \in \mathbb{Z}^{v} \mid n_{v} \geq 1\right\}$.

(iii) If one uses Neumann boundary conditions (i.e., restriction of a whole-line even operator to even functions), then weak coupling negative $b_{n}$ 's and $a_{n} \equiv 1$ produce a bound state (see, e.g., Simon [4]) and so the analog of Corollary 1.4, or even Theorem 1.2, fails.

I would like to thank Alexander Aptekarev, David Damanik, Rowan Killip, Rostyslav Kozhan, and Sasha Pushnitski for useful correspondence and/or discussions.

\section{The discrete case}

In this section, we will prove Theorem 1.3. We begin with the following lemma.

Lemma 2.1. Let $P_{n}$ be a family of orthogonal projections on a Hilbert space $\mathscr{H}$ with

$$
\operatorname{s-lim}_{n \rightarrow \infty} P_{n}=1
$$

Let $J$ be a bounded selfadjoint operator on $\mathscr{H}$ so that

$$
\operatorname{dim} P_{(-\infty, 0)}(J)=\ell<\infty,
$$

where $P_{(-\infty, 0)}(\cdot)$ is a spectral projection. Then, for some $N$,

$$
n>N \Longrightarrow \operatorname{dim} P_{(-\infty, 0)}\left(P_{n} J P_{n}\right)=\ell .
$$

Proof. By a variational argument,

$$
\operatorname{dim} P_{(-\infty, 0)}\left(P_{n} J P_{n}\right) \leq \ell
$$


(essentially, if it were dimension $\geq \ell+1$, we could find $\ell+1$ unit vectors in $\operatorname{Ran} P_{n}$ so that $\left\langle\varphi_{j}, \varphi_{m}\right\rangle=\delta_{j m}$ and $\left\langle\varphi_{j}, J \varphi_{m}\right\rangle=a_{j} \delta_{j m}$ with $\left.a_{j}<0\right)$.

For any $\varphi_{1}, \ldots, \varphi_{\ell}$ in $\operatorname{Ran} P_{n}$, let $\Delta_{j k}=\left\langle\varphi_{j}, \varphi_{k}\right\rangle$ and $A_{j k}=\left\langle\varphi_{j}, J \varphi_{k}\right\rangle$. If $\Delta$ is invertible and $\Delta^{-1 / 2} A \Delta^{-1 / 2}<0$, then (9) holds by the variational principle.

Let $\psi_{1}, \ldots, \psi_{\ell}$ span $\operatorname{Ran} P_{(-\infty, 0)}(J)$ and be a set of orthonormal eigenvectors for $J$. Let $\varphi_{j}^{(n)}=P_{n} \psi_{j}$ and $\Delta_{j k}^{(n)}=\left\langle\varphi_{j}^{(n)}, \varphi_{k}^{(n)}\right\rangle, A_{j k}^{(n)}=\left\langle\varphi_{j}^{(n)}, J \varphi_{k}^{(n)}\right\rangle$. By (6), $\Delta_{j k}^{(n)} \rightarrow \mathbf{1}, A_{j k}^{(n)} \rightarrow \alpha_{j} \delta_{j k}$ with $\alpha_{j}<0$. It follows for all large $n$ that $\Delta^{(n)}$ is invertible and $\left(\Delta^{(n)}\right)^{-1 / 2} A^{(n)}\left(\Delta^{(n)}\right)^{-1 / 2}<0$.

Proof of Theorem 1.3. Let $S_{n}=P_{n}+Q_{n}$. Then, by a variational argument,

$$
\operatorname{dim} P_{(-\infty, 0)}\left(S_{n} J S_{n}\right) \leq \operatorname{dim} P_{(-\infty, 0)}(J) \equiv \ell<\infty .
$$

By (5), on $\operatorname{Ran} S_{n}=\operatorname{Ran} P_{n} \oplus \operatorname{Ran} Q_{n}$, we have that

$$
S_{n} J S_{n}=P_{n} J P_{n} \oplus Q_{n} J Q_{n}
$$

so

$\operatorname{dim} P_{(-\infty, 0)}\left(S_{n} J S_{n}\right)=\operatorname{dim} P_{(-\infty, 0)}\left(P_{n} J P_{n}\right)+\operatorname{dim} P_{(-\infty, 0)}\left(Q_{n} J Q_{n}\right)$.

By the lemma for some $N$ and $n>N$,

$$
\operatorname{dim} P_{(-\infty, 0)}\left(P_{n} J P_{n}\right)=\ell
$$

By (10), (12), and (13) for $n>N$,

$$
\operatorname{dim} P_{(-\infty, 0)}\left(Q_{n} J Q_{n}\right)=0
$$

that is, $Q_{n} J Q_{n} \geq 0$.

\section{The continuum case}

Here we will state and prove the analog of Theorem 1.3 for ODEs. For simplicity of exposition, we assume the potential is bounded, but it is easy to extend to $V$ 's with $\|V(x)\|$ in $L_{\text {loc }}^{1}$ with some kind of selfadjointness criterion at $\infty$ or to positive $V$ 's using quadratic forms.

Theorem 3.1. Let $\mathcal{K}$ be a Hilbert space and $V(x), x \in[0, \infty)$, a weakly measurable function on $[0, \infty)$ with values in the bounded operators on $\mathcal{K}$ with $\sup _{x \in[0, \infty)}\|V(x)\|$ $<\infty$. Let $H_{a}, a \geq 0$, be the operator on $\mathscr{H}_{a} \equiv L^{2}((a, \infty), \mathcal{K} ; d x)$ of the form $-\frac{d^{2}}{d x^{2}}+V(x)$ with $u(a)=0$ boundary conditions. Suppose $H \equiv H_{a=0}$ has only finitely many eigenvalues in $(-\infty, 0)$, each of finite multiplicity and no other spectrum there. Then for some $A \in[0, \infty), H_{a} \geq 0$ for $a \geq A$. 
Remark 3.2. By allowing $V(x)$ to be a selfajdoint operator bounded from below (to handle the Laplacian in the other directions), this theorem can be extended to treat $-\Delta+V\left(x_{1}, \ldots, x_{v}\right)$ on $\left\{x \mid x_{v}>0\right\}$.

Proof. For each $a$, by adding a Dirichlet boundary condition at $a$, we can find $\widetilde{H}_{a}$ on $\mathscr{H}_{a=0}$ of the form $K_{a} \oplus H_{a}$ on $L^{2}((0, a), \mathcal{K} ; d x) \oplus L^{2}((a, \infty), \mathcal{K} ; d x)$ and

$$
\tilde{H}_{a} \geq H_{a=0} .
$$

As in the proof of Lemma 2.1,

$$
\operatorname{dim} P_{(-\infty, 0)}\left(K_{a}\right)=\operatorname{dim} P_{(-\infty, 0)}\left(H_{a=0}\right)
$$

for all $a>A$ for some $A$.

For such $a$, by (14), $\operatorname{dim} P_{(-\infty, 0)}\left(H_{a}\right)=0$.

\section{References}

[1] M. Bôcher, The theorems of oscillation of Sturm and Klein. Bull. Amer. Math. Soc. 4 (1897-1898), 295-313, 365-376. Jmf 29.0285.01

[2] E. M. Nikishin, The discrete Sturm-Liouville operator and some problems of function theory. J. Soviet Math. 35 (1986), 2679-2744.

[3] H. Schulz-Baldes, Rotation numbers for Jacobi matrices with matrix entries. Math. Phys. Electron. J. 13 (2007), Paper 5, 40 pp. (electronic). MR 2366118

[4] B. Simon, The bound state of weakly coupled Schrödinger operators in one and two dimensions. Ann. Phys. 97 (1976), 279-288. MR 0404846 Zbl 1141.15024

[5] B. Simon, Sturm oscillation and comparison theorems. In Sturm-Liouville Theory: Past and Present (W. Amrein, A. Hinz and D. Pearson. eds.), pp. 29-43, Birkhäuser, Basel, 2005. MR 2145076 Zbl 1117.39013

Received July 1, 2010, revised August 26, 2010

Barry Simon, Mathematics 253-37, California Institute of Technology, Pasadena, CA 91125, U.S.A.

E-mail: bsimon@caltech.edu 